\title{
A NOTE ON THE RECIPROCAL OF A FOURIER SERIES
}

\section{A. EDREI AND G. SZEGÖ}

Introduction. Let $f(\theta)$ be a real function which belongs to the class $L(0,2 \pi)$ and let

$$
\sum_{\nu=-\infty}^{+\infty} a_{\nu} e^{i \nu \theta}
$$

be its complex Fourier series.

If $[f(\theta)]^{-1}$ also belongs to $L(0,2 \pi)$, we may consider its Fourier series

$$
\sum_{\nu=-\infty}^{+\infty} b_{\nu} e^{i v \theta}
$$

and investigate the connection between the Fourier coefficients of $f(\theta)$ and $[f(\theta)]^{-1}$.

Formal multiplication of (1) and (2) yields the infinite system

(3) $\sum_{\nu=-\infty}^{+\infty} a_{\mu-\nu} b_{\nu}=\delta_{0 \mu}\left(\mu=0, \pm 1, \pm 2, \cdots ; \delta_{00}=1 ; \delta_{0 \mu}=0\right.$ if $\left.\mu \neq 0\right)$,

which it is natural to attempt to solve by the "method of sections" (that is the "méthode des réduites" discussed by F. Riesz [1, p. 2]). Let

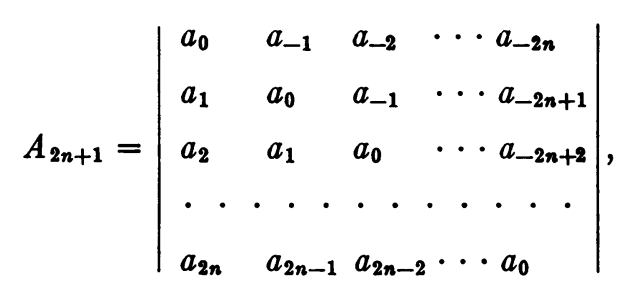

and denote by $A_{2 n+1}(j, k)$ the minor obtained by deleting the $(n+1+j)$ th row and the $(n+1+k)$ th column of $A_{2 n+1}$. The formal method just mentioned will be justified as soon as we establish the relation $^{1}$

$$
\lim _{n \rightarrow \infty}(-1)^{j-k} \frac{A_{2 n+1}(j, k)}{A_{2 n+1}}=b_{k-j}
$$

Received by the editors May 23, 1952.

${ }^{1}$ Strictly speaking, the "methode des réduites" requires only the proof of the special case $j=0$, of the relation (4). 
Indeed, we prove the following

Theorem. Let $f(\theta)$ be a real function defined in the interval $(0,2 \pi)$, and assume

(i) $0 \leqq f(\theta)$;

(ii) $f(\theta) \in L$;

(iii) $[f(\theta)]^{-1} \in L$.

If (1) and (2) denote the respective complex Fourier series of $f(\theta)$ and $[f(\theta)]^{-1}$, we have

$$
A_{2 n+1}>0 \text {, }
$$

and the relation (4) is true for all fixed ${ }^{2}$ integral values of $j$ and $k$.

The inequality (5) is not new (cf. [3, p. 189]). It is easily proved under the assumptions

$$
0 \leqq f(\theta) ; \quad f(\theta) \in L ; \quad \int_{0}^{2 \pi} f(\theta) d \theta>0 .
$$

The relation (4) was noticed and proved by Edrei under the assumptions $^{3}$

(I) $0 \leqq f(\theta) \leqq M<+\infty$;

(II) $f(\theta) \in L$;

(III) $[f(\theta)]^{-1} \in L^{2}$;

his proof is given in $\$ 2$.

The final form of the result is due to Szegö; his proof is given in $\$ 3$

1. Formal observations. We consider the trigonometric polynomial

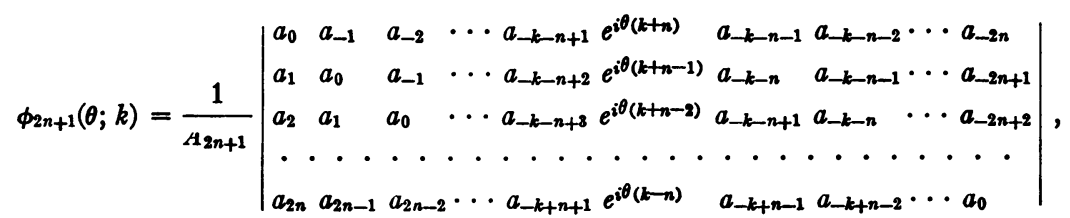

and the determinant

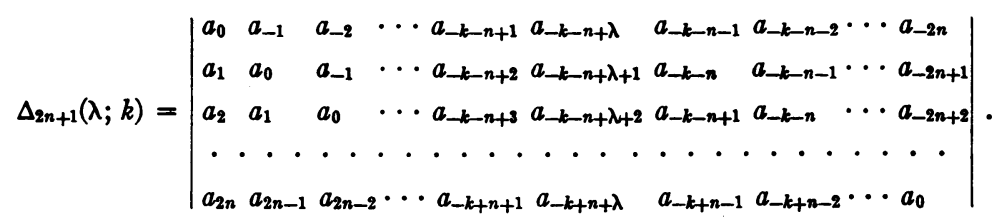

2 The proof of this theorem, to be given in $\$ 3$, furnishes in fact more, namely the relation (4) with the following interpretation: the numbers $j$ and $k$ are not necessarily fixed; however, they depend on $n$ in such a way that $j-k=$ const. and $n-|j| \rightarrow \infty$.

3 Even in this incomplete form, the result lends itself to applications. One of the authors of this paper uses it as an essential tool in the proof which he recently obtained of a conjecture of Schoenberg on the generating function of a totally positive sequence (cf. $[2$, p. 362$]$ ). 
Obviously, for $-k-n \leqq \lambda \leqq-k+n$,

$$
\frac{1}{2 \pi} \int_{0}^{2 \pi} \phi_{2 n+1}(\theta ; k) e^{i \lambda 0} d \theta=(-1)^{\lambda} \frac{A_{2 n+1}(k+\lambda, k)}{A_{2 n+1}},
$$

and for any integer $\lambda$,

$$
\frac{1}{2 \pi} \int_{0}^{2 \pi} \phi_{2 n+1}(\theta ; k) e^{-i \lambda} f(\theta) d \theta=\frac{\Delta_{2 n+1}(\lambda ; k)}{A_{2 n+1}} .
$$

Noticing that

$$
\begin{aligned}
& \Delta_{2 n+1}(\lambda ; k)=0 \quad \text { for } \lambda=-n+k,-n+k+1, \cdots,-2,-1, \\
& \Delta_{2 n+1}(0 ; k)=A_{2 n+1}, \\
& \Delta_{2 n+1}(\lambda ; k)=0 \quad \text { for } \lambda=1,2,3, \cdots, n+k,
\end{aligned}
$$

we see that (1.2) implies

$$
\frac{1}{2 \pi} \int_{0}^{2 \pi}\left|\phi_{2 n+1}(\theta ; k)\right|^{2} f(\theta) d \theta=\frac{A_{2 n+1}(k, k)}{A_{2 n+1}} .
$$

Now

$$
\begin{aligned}
0 \leqq & \frac{1}{2 \pi} \int_{0}^{2 \pi} \frac{\left|1-f(\theta) \phi_{2 n+1}(\theta ; k)\right|^{2}}{f(\theta)} d \theta \\
= & b_{0}+\frac{1}{2 \pi}\left\{\int_{0}^{2 \pi} f(\theta)\left|\phi_{2 n+1}(\theta ; k)\right|^{2} d \theta\right. \\
& -\int_{0}^{2 \pi} \phi_{2 n+1}(\theta ; k) d \theta \\
& -\int_{0}^{2 \pi} \frac{\left.\phi_{2 n+1}(\theta ; k) d \theta\right\}}{}
\end{aligned}
$$

which, in view of (1.1) and (1.4), yields

$$
\frac{1}{2 \pi} \int_{0}^{2 \pi}\left|\phi_{2 n+1}(\theta ; k)\right|^{2} f(\theta) d \theta \leqq b_{0} .
$$

2. Study of a special case. If we replace the assumptions (i), (ii), and (iii) of the theorem by the more restrictive conditions (I), (II), and (III), we may apply Parseval's theorem to the left-hand sides of (1.1) and (1.2). We obtain respectively,

$$
\frac{1}{2 \pi} \int_{0}^{2 \pi}\left\{\phi_{2 n+1}(\theta ; k) e^{i \lambda \theta} f(\theta)\right\} \frac{1}{f(\theta)} d \theta=\sum_{n=-\infty}^{+\infty} \frac{\Delta_{2 n+1}(\nu-\lambda ; k)}{A_{2 n+1}^{2}} b_{-\nu}
$$


and

$$
\frac{1}{2 \pi} \int_{0}^{2 \pi}\left|\phi_{2 n+1}(\theta ; k)\right|^{2} f^{2}(\theta) d \theta=\sum_{\nu=-\infty}^{+\infty} \frac{\left|\Delta_{2 n+1}(\nu ; k)\right|^{2}}{A_{2 n+1}^{2}} .
$$

By assumption (I),

$$
\int_{0}^{2 \pi}\left|\phi_{2 n+1}(\theta ; k)\right|^{2} f^{2}(\theta) d \theta \leqq M \int_{0}^{2 \pi}\left|\phi_{2 n+1}(\theta ; k)\right|^{2} f(\theta) d \theta,
$$

so that (2.2) and (1.5) imply

$$
\sum_{n=-\infty}^{+\infty} \frac{\left|\Delta_{2 n+1}(\nu ; k)\right|^{2}}{A_{2 n+1}^{2}} \leqq M b_{0} .
$$

Now (2.1), (1.1), and (1.3) yield

$$
\begin{aligned}
(-1)^{j-k} \frac{A_{2 n+1}(j, k)}{A_{2 n+1}}-b_{k-j}= & \sum_{\nu=-\infty}^{-n+j-1} \frac{\Delta_{2 n+1}(\nu-j+k ; k)}{A_{2 n+1}} b_{\rightarrow} \\
& +\sum_{\nu=n+j+1}^{+\infty} \frac{\Delta_{2 n+1}(\nu-i+k ; k)}{A_{2 n+1}} b_{\rightarrow \cdot}
\end{aligned}
$$

In view of (2.3) and of the convergence of $\sum\left|b_{\nu}\right|^{2}$, the right-hand side of (2.4) may be estimated by Schwarz's inequality; we find

$$
\begin{aligned}
\mid(-1)^{j-k} \frac{A_{2 n+1}(j ; k)}{A_{2 n+1}} & -b_{k-j} \mid \\
& \leqq\left(M b_{0}\right)^{1 / 2}\left\{\sum_{n=-\infty}^{-n+j-1}\left|b_{-v}\right|^{2}+\sum_{v=n+j+1}^{\infty}\left|b_{-}\right|^{2}\right\}^{1 / 2} .
\end{aligned}
$$

Letting $n \rightarrow \infty$, we obtain (4).

3. Proof of the theorem. In view of (1.1), our theorem may be proved by showing that the polynomials $\phi_{2 n+1}(\theta ; k)$ converge weakly to $[f(\theta)]^{-1}$, that is

$$
\begin{aligned}
& \lim _{n \rightarrow \infty} \int_{0}^{2 \pi}\left\{\phi_{2 n+1}(\theta ; k)-\frac{1}{f(\theta)}\right\} e^{i \lambda \theta} d \theta=0 \\
&(\lambda=0, \pm 1, \pm 2, \cdots) .
\end{aligned}
$$

Let $h(\theta)$ be an arbitrary trigonometric polynomial. The obvious identity

$$
\phi_{2 n+1}-1 / f=\left(f \phi_{2 n+1}-1\right) h+(1-h f) \phi_{2 n+1}+(h-1 / f)
$$

implies 


$$
\begin{aligned}
& \left|\int_{0}^{2 \pi}\left\{\phi_{2 n+1}(\theta ; k)-\frac{1}{f(\theta)}\right\} e^{2 \lambda \theta} d \theta\right| \\
(3.2) & \leqq \\
& +\int_{0}^{2 \pi}\left\{f(\theta) \phi_{2 n+1}(\theta ; k)-1\right\} h(\theta) e^{2 \lambda \theta} d \theta \mid \\
& |1-h(\theta) f(\theta)|\left|\phi_{2 n+1}(\theta ; k)\right| d \theta+\int_{0}^{2 \pi}\left|h(\theta)-\frac{1}{f(\theta)}\right| d \theta
\end{aligned}
$$

By Schwarz's inequality

$$
\begin{aligned}
\left\{\int_{0}^{2 \pi} \frac{|h(\theta) f(\theta)-1|}{(f(\theta))^{1 / 2}} \frac{1}{(f(\theta))^{1 / 2}} d \theta\right\}^{2} & \\
& \leqq 2 \pi b_{0} \int_{0}^{2 \pi} \frac{|h(\theta) f(\theta)-1|^{2}}{f(\theta)} d \theta
\end{aligned}
$$

and, as a further consequence of Schwarz's inequality and (1.5), we obtain

$$
\begin{aligned}
&\left\{\int_{0}^{2 \pi} \frac{|1-h(\theta) f(\theta)|}{(f(\theta))^{1 / 2}}(f(\theta))^{1 / 2}\left|\phi_{2 n+1}(\theta ; k)\right| d \theta\right\}^{2} \\
& \leqq 2 \pi b_{0} \int_{0}^{2 \pi} \frac{|1-h(\theta) f(\theta)|^{2}}{f(\theta)} d \theta
\end{aligned}
$$

We now choose $h(\theta)$ so that

$$
J[h(\theta)]=\int_{0}^{2 \pi} \frac{|1-h(\theta) f(\theta)|^{2}}{f(\theta)} d \theta
$$

is small.

Let $\epsilon_{1}$ and $\epsilon_{2}$ be positive parameters and consider the following three sets of points (of the interval $[0,2 \pi]$ ):

$E_{1}=E\left(f(\theta) \leqq \epsilon_{1}\right) ; \quad E_{2}=E\left(\epsilon_{1}<f(\theta) \leqq \epsilon_{2}^{-1}\right) ; \quad E_{2}=E\left(\epsilon_{2}^{-1}<f(\theta)\right)$.

Moreover, denote by $F(\theta)$ a function which is equal to $[f(\theta)]^{-1}$ on $E_{2}$ and vanishes on both $E_{1}$ and $E_{3}$. Then

$$
0 \leqq F(\theta)<\epsilon_{1}^{-1},
$$

so that, given $\epsilon(>0)$, there exists a trigonometric polynomial ${ }^{4} h(\theta)$ :

\footnotetext{
$h(\theta)$.

4 The Fejer-means of the Fourier series of $F(\theta)$ satisfy the conditions imposed on
} 


$$
h(\theta)=\sum_{n=l}^{+l} h_{\nu} e^{i \nu \theta} \quad\left(h_{-\nu}=\overline{h_{\nu}}\right),
$$

such that

$$
\begin{gathered}
\int_{0}^{2 \pi}\{F(\theta)-h(\theta)\}^{2} d \theta<\epsilon, \\
0 \leqq h(\theta) \leqq \epsilon_{1}^{-1} .
\end{gathered}
$$

Using (3.5), (3.6), the mean-value theorem, and the definitions of $E_{1}, E_{2}$, and $E_{3}$, we obtain

$$
\begin{aligned}
\int_{E_{2}} \frac{\{1-h(\theta) f(\theta)\}^{2}}{f(\theta)} d \theta & =\int_{E_{2}} f(\theta)\{F(\theta)-h(\theta)\}^{2} d \theta \leqq \epsilon_{2} \epsilon \\
\int_{E_{1}} \frac{\{1-h(\theta) f(\theta)\}^{2}}{f(\theta)} d \theta & \leqq \int_{E_{1}} \frac{d \theta}{f(\theta)}+\int_{B_{1}} h^{2}(\theta) f(\theta) d \theta \\
& =\int_{E_{1}} \frac{d \theta}{f(\theta)}+\int_{E_{1}}\{F(\theta)-h(\theta)\}^{2} f(\theta) d \theta \\
& \leqq \int_{E_{1}} \frac{d \theta}{f(\theta)}+\epsilon_{1} \epsilon_{1} \\
\int_{E_{3}} \frac{\{1-h(\theta) f(\theta)\}^{2}}{f(\theta)} d \theta & \leqq \int_{E_{3}} \frac{d \theta}{f(\theta)}+\int_{E_{3}} h^{2}(\theta) f(\theta) d \theta \\
& \leqq 2 \pi \epsilon_{2}+\bar{\epsilon}_{1}^{2} \int_{E_{3}} f(\theta) d \theta .
\end{aligned}
$$

Taking

$$
\epsilon_{2}=\epsilon^{1 / 2} ; \quad \epsilon_{1}(\epsilon)=\left\{\int_{E_{3}} f(\theta) d \theta\right\}^{1 / 4},
$$

the inequalities (3.7) yield

$$
J[h(\theta)] \leqq(1+2 \pi) \epsilon^{1 / 2}+\epsilon_{1} \epsilon+\int_{B_{1}} \frac{d \theta}{f(\theta)}+\left\{\int_{E_{3}} f(\theta) d \theta\right\}^{1 / 2} .
$$

Hence, given $\delta(>0)$, it is always possible to determine a trigonometric polynomial $h(\theta)$, of degree $l(\delta)$, such that

$$
J[h(\theta)]<\delta .
$$

In view of (1.2) and (1.3) the first term of the right-hand side of (3.2) vanishes as soon as

$$
n \geqq l(\delta)+|k+\lambda|,
$$


so that the relation (3.1) is an obvious consequence of (3.2), (3.3), (3.4), and (3.8).

\section{REFERENCES}

1. F. Riesz, Les systèmes d'équations lineaires d une infinité d'inconnues, Paris, 1913.

2. I. J. Schoenberg, Some analytical aspects of the problem of smoothing, Courant Anniversary Volume, New York, 1948, pp. 351-370.

3. G. Szegö, Beiträge zur Theorie der Toeplitzschen Formen, Math. Zeit. vol. 6 (1920) pp. 167-202.

UNIVERSITY OF Colorado AND

STANFORD UNIVERSITY

\section{A NEUMANN SERIES FOR THE PRODUCT OF TWO WHITTAKER FUNCTIONS'}

\section{PETER HENRICI}

1. Introduction. Several series representations in terms of Bessel functions have been given recently for a single Whittaker function of the first kind (usually denoted by $M_{k, \mu}(z)$ ) by various authors. ${ }^{2}$ None of these series is very easy to survey, because their coefficients are never given explicitly but only either by means of a generating function (as in [4]) or by recurrence formulae (as in [1] and [6]). In the present paper a generalized Neumann series for the product of two Whittaker functions of the first kind with common indices but different arguments is given, in which the coefficients are formed by certain terminating generalized hypergeometric series and by Gegenbauer polynomials. The expansion obtained includes also the case of a single Whittaker function, which is of special interest in connection with the theory of Coulomb waves. ${ }^{3}$ Our method of proof uses only the simplest properties of the function $M_{x, \mu}(z)$ and is especially much more elementary than the methods of [4]. All symbols used are those of Magnus-Oberhettinger [7], with exception of that for the generalized hypergeometric series, for which we use Bailey's [2] notation.

2. Theorem. For arbitrary complex values or $r, \theta, k$, and $4 \mu$ $\neq-1,-2, \cdots$ the following expansion holds:

Received by the editors May 26, 1952.

1 This paper was prepared in part under a National Bureau of Standards Contract with The American University.

2 Abramowitz [1], Buchholz [4], Karlin [6], H. Schmidt [4], Tricomi [4].

${ }^{3} \mathrm{Cf}$. [1] and the literature quoted there. The function $\phi_{L}(\eta, \rho)$ considered in [1] is identical with $M_{i m L+1 / 2}(2 i \rho) \cdot(2 i \rho)^{-(L+1)}$. 